\title{
Growth inhibition of colon cancer cells by transfection of dominant-negative apoptosis signal-regulating kinase-1
}

\author{
HIKARU KUWAMURA ${ }^{1,2}$, KAZUNARI TOMINAGA ${ }^{1}$, MASAYUKI SHIOTA $^{2}$, REIKO ASHIDA ${ }^{1}$, \\ TAKAFUMI NAKAO $^{2}$, EIJI SASAKI ${ }^{1}$, TOSHIO WATANABE ${ }^{1}$, YASUHIRO FUJIWARA $^{1}$, \\ NOBUHIDE OSHITANI ${ }^{1}$, KAZUHIDE HIGUCHI ${ }^{1}$, HIDENORI ICHIJO ${ }^{3}$, \\ TETSUO ARAKAWA ${ }^{1}$ and HIROSHI IWAO ${ }^{2}$
}

\begin{abstract}
Departments of ${ }^{1}$ Gastroenterology and ${ }^{2}$ Pharmacology, Osaka City University Graduate School of Medicine, Osaka; ${ }^{3}$ Laboratory of Cell Signaling, Graduate School of Pharmaceutical Sciences, University of Tokyo, CREST, Tokyo, Japan
\end{abstract}

Received November 30, 2006; Accepted January 12, 2007

\begin{abstract}
Apoptosis signal-regulating kinase-1 (ASK-1) is an important molecule for the pro-apoptotic signaling. ASK-1 also contributes to the cellular survival for many types of cells. Thus, ASK-1 has a broad range of biological activities depending on the cell type. The present study assessed the role(s) of ASK-1 in colorectal cancer cells (HT-29) by using adenovirus vectors expressing wild-type (WT)-ASK-1 or dominant-negative (DN) mutant of ASK-1 and recombinant adenovirus containing the bacterial $B$-galactosidase gene (Ad-LacZ), a negative control for Ad-DN-ASK-1. Selective phosphorylation of ASK-1 at Thr 845, a kinase domain site, but not Ser 83 nor 967 sites was induced by serum stimulation in a time-dependent manner. Transfection with Ad-DNASK-1 inhibited the serum-induced phosphorylation of $\mathrm{p} 38$ mitogen-activated protein kinase, a downstream molecule of ASK-1. Transfection with Ad-DN-ASK-1 diminished the serum-induced cell proliferation in a dose-dependent manner, whereas WT-ASK-1 increased it. Apoptosis assessed by Hoechst staining was induced in the Ad-DN-ASK-1 treated cells. In vivo transfection of Ad-DN-ASK-1 into tumor xenografts of HT-29 cells in nude mice significantly decreased the tumor volume on day 29. Cleaved caspase-3 was found in the tumors of DN-ASK-1 treated mice. We obtained the first evidence that DN-ASK-1 transfection exerted significant antitumor effects on colon cancer mediated by apoptosis.
\end{abstract}

Correspondence to: Dr Kazunari Tominaga, Department of Gastroenterology, Graduated School of Medicine, Osaka City University Medical School, 1-4-3 Asahimachi, Abeno-ku, Osaka 545-8585, Japan

E-mail: tomy@med.osaka-cu.ac.jp

Key words: apoptosis signal-regulating kinase-1, gene therapy, transfection, colon cancer, apoptosis, caspase-3

\section{Introduction}

Apoptosis signal-regulating kinase-1 (ASK-1) is expressed in various types of cells and tissues (1). A variety of external stimuli such as chemical, mechanical, or oxidative stresses and proinflammatory cytokines such as tumor necrosis factor (TNF) or interleukin (IL)-1ß induced the activation of ASK-1, followed by the activation of c-Jun NH2-terminal kinases (JNK) and p38 mitogen-activated protein kinase (MAPK) (2-6). ASK-1-p38 MAPK/JNK cascade is associated with apoptosis of various types of cultured cells (7). Apoptosis is significantly inhibited in fibroblasts derived from knockout mouse fetus of ASK-1 (8). ASK-1 has thus been generally considered to induce apoptosis. However, it has been reported that pathway(s) activating p38 via the activation of ASK-1 induce the survival of epithelial cells and nondifferentiated neuronal cells $(9,10)$. Previous evidence has suggested that ASK-1 not only induces apoptosis, but also regulates the cell cycle. The biological properties of ASK-1 may depend on the cell type or conditions. Furthermore, the role of ASK-1 in cellular proliferation remains unclear.

Accumulating evidence has demonstrated that the pathogenesis of colon cancer involves multiple, progressive steps associated with a number of genetic alterations, including mutations of tumor suppressor genes and proto-oncogenes, such as APC, K-ras, p53, or DCC1 (11). Basic molecular mechanisms underlying colon cancer growth have been studied extensively, with the ultimate goal of delineating critical targets for the development of novel treatments. We have shown that the transcription factor activator protein-1 (AP-1) is essential for the growth of colon cancer (HT-29 cells). We obtained evidence that dominant-negative (DN)-c-Jun gene transfer significantly inhibits the growth of colon cancer (12).

The pathway from the activation of ASK-1 followed by that of JNK and/or p38 MAPK has been reported to reside upstream of the transcription of AP-1 (13). Available evidence thus suggests that elucidation of the role of ASK-1 in the proliferation of colon cancer cells may be critical to understanding the pathogenesis of colon cancer and establishing new strategies for treatment. 
In the present study, we constructed a recombinant adenovirus containing the dominant-negative mutant of ASK-1 (Ad-DN-ASK-1) and transfected it into colon cancer cells (HT-29) in vitro and in vivo to elucidate the roles of ASK-1. We found that inhibition of the ASK-1-p38 MAPK pathway inhibited HT-29 cell proliferation mediated by inducing apoptosis. Our results provide initial evidence that Ad-DNASK-1 transfection significantly inhibits the growth of colon cancer cells and may thus be used as a therapeutic strategy for colon cancer.

\section{Materials and methods}

Cells and culture conditions. The human colon adenocarcinoma cell line HT-29 was purchased from American Type Culture Collection. Cells were continuously grown as monolayers in McCoy's 5A medium, supplemented with $10 \%$ heat-inactivate fetal bovine serum (FBS) in an atmosphere of $5 \% \mathrm{CO}_{2}$ and $95 \% \mathrm{O}_{2}$ at $37^{\circ} \mathrm{C}$. The cells were subcultured every 7 days.

Construction of recombinant adenovirus containing the dominant-negative mutant of ASK-1 (Ad-DN-ASK1). Adenovirus vectors expressing wild-type (WT)-ASK-1 (AdWT-ASK-1) and dominant-negative mutant of ASK-1 (AdDN-ASK-1)(Lys 709 to Met), tagged hemagglutinin (HA) in the site of $\mathrm{NH}_{2}$-terminus, have been described previously (3). Recombinant adenovirus containing the bacterial $B$-galactosidase gene (Ad-LacZ) was constructed as a negative control for Ad-DN-ASK-1. The titer of the virus was determined by Adeno- $\mathrm{X}^{\mathrm{TM}}$ rapid titer kit (Clontech, USA) and expressed in i.f.u.

Adenovirus-mediated gene transfer to HT-29 cells in vitro. In vitro gene transfer to HT-29 cells was carried out by incubation with the adenoviral vector with MOI of 30-100 in McCoy's 5A medium for $1 \mathrm{~h}$, followed by addition of $1 \%$ FBS overnight at $37^{\circ} \mathrm{C}$ in an atmosphere of $5 \% \mathrm{CO}_{2}$ and $95 \%$ $\mathrm{O}_{2}$. HT-29 cells were then made quiescent for $24 \mathrm{~h}$ before the experiments.

Measurement of cell proliferation. For cell proliferation assay, HT-29 cells, infected with Ad-LacZ, Ad-WT-ASK-1, or Ad-DN-ASK-1 at 30, 50, or $100 \mathrm{MOI}$ in six-well cultured plates, were stimulated by $1 \%$ FBS for 5 days and harvested by trypsin/EDTA. Viable cell number was counted by a coulter counter (Beckman Coulter).

Western blotting. Cells were washed and then dissolved in lysis buffer (50 mM HEPES [pH 8.0], $150 \mathrm{mM} \mathrm{NaCl}, 1 \%$ [vol/vol] Triton-X100, 10\% [vol/vol] glycerol, $25 \mathrm{mM}$ EDTA, complete protease inhibitor cocktail tablet [Roche]). Lysates were clarified by centrifugation at $13,500 \mathrm{x} \mathrm{g}, 4^{\circ} \mathrm{C}$. Lysates for immunoblotting (30 $\mu \mathrm{g}$ of protein) were separated on 7.5 or $12.5 \%$ SDS-polyacrylamide gels under reducing conditions, followed by electrophoretic transfer to polyvinylidine difluoride membranes (Immobilon-P, Millipore Corp., Bedford, MA). After blocking with 5\% BSA in TBS-T for a 1-h incubation, the membranes were incubated with the relevant primary antibodies in the same buffer for $1 \mathrm{~h}$. The membranes were washed in TBS-T 3 times and further incubated with horseradish peroxidase-linked secondary antibodies (1:5000) (Amersham Life Sciences Inc., Buckinghamshire, UK). After washing for another hour, the blots were developed using an enhanced chemiluminescence system (Amersham) and LAS1000 plus (Fujifilm, Tokyo, Japan). Anti- $\alpha$-tublin (Oncogene) and $\beta$-actin (Sigma, USA) were used at 1:5000 dilutions. Anti-cleaved-caspase-3 (Abcom, UK), HA-7 (Sigma), anti-ASK-1, anti-phospho-ASK-1 (Ser83), anti-phospho-ASK-1 (Ser-967), anti-phospho-ASK-1 (Thr-845), and anti-phospho-p38 mitogen activated protein kinase (MAPK) (all from Cell Signaling Technology, Beverly, MA) were used at dilutions of 1:1000.

Hoechst 33342 staining. Detached and adsorbed cells were washed with PBS and fixed in $4 \%$ (wt/vol) paraformaldehyde at $4^{\circ} \mathrm{C}$ overnight. Thereafter, the cells were washed with PBS, stained with $1.6 \mu \mathrm{M}$ Hoechst 33342 (Calbiochem, USA) for $10 \mathrm{~min}$, resuspended, seeded on glass slides, and examined by fluorescence microscopy.

Production of xenografted tumor of HT-29 cells and treatment with $A d-D N-A S K 1$. HT-29 cells ( $1 \times 10^{6}$ cells) were inoculated subcutaneously into the hind paws of BALB/cA Jcl nu/nu female mice (4-5 weeks old; CLEA Japan, Inc., Osaka, Japan). After reaching a mean volume of $150 \mathrm{~mm}^{3}$, the tumors were randomly assigned to three groups in a blinded manner; group 1, Ad-DN-ASK-1 (3x10 $10^{8}$ i.f.u.); group 2, Ad-LacZ ( $3 \times 10^{8}$ i.f.u.); and group 3 , control vehicle (PBS) $(n=8)$. Direct injection was performed 3 times a week, for a total of six sessions. Each injection of purified virus was diluted in a total volume of $30 \mu \mathrm{l}$ PBS and administered in a single pass with a 30 -gauge needle, using gentle, constant infusion pressure during anesthesia with diethylether. Tumors were measured 3 times per week immediately before each treatment. Tumor size was estimated using the following formula: length $(\mathrm{mm}) \mathrm{x}$ width $(\mathrm{mm}) \mathrm{x}$ width $(\mathrm{mm}) / 2$. All experimental procedures were approved by the Animal Care Committee of Osaka City University Medical School.

Statistical analysis. The values are presented as means \pm SEM. Statistical significance was determined with one-way analysis of variance and Duncan's multiple range test. Differences were considered statistically significant at a value of $\mathrm{p}<0.05$.

\section{Results}

Phosphorylation of ASK-1 in HT-29 cells stimulated by FBS (1\%). Activation of ASK-1 phosphorylation requires threonine residue (Thr 845), which is present in the activation loop of the kinase domain. Phosphorylation of ASK-1 at Thr 845, but not at sites Ser 83 nor 967, was induced in HT-29 cells by serum stimulation in a time-dependent manner. By $30 \mathrm{~min}$, phosphorylation levels were maximal as compared with the control levels (Fig. 1).

Efficiency of ASK-1 gene transfer for p38 MAPK phosphorylation. After infection with Ad-LacZ at MOI of 3, 30, or 100, the efficiency of gene transfer by our previously reported transfection techniques into HT-29 cells was reconfirmed by 


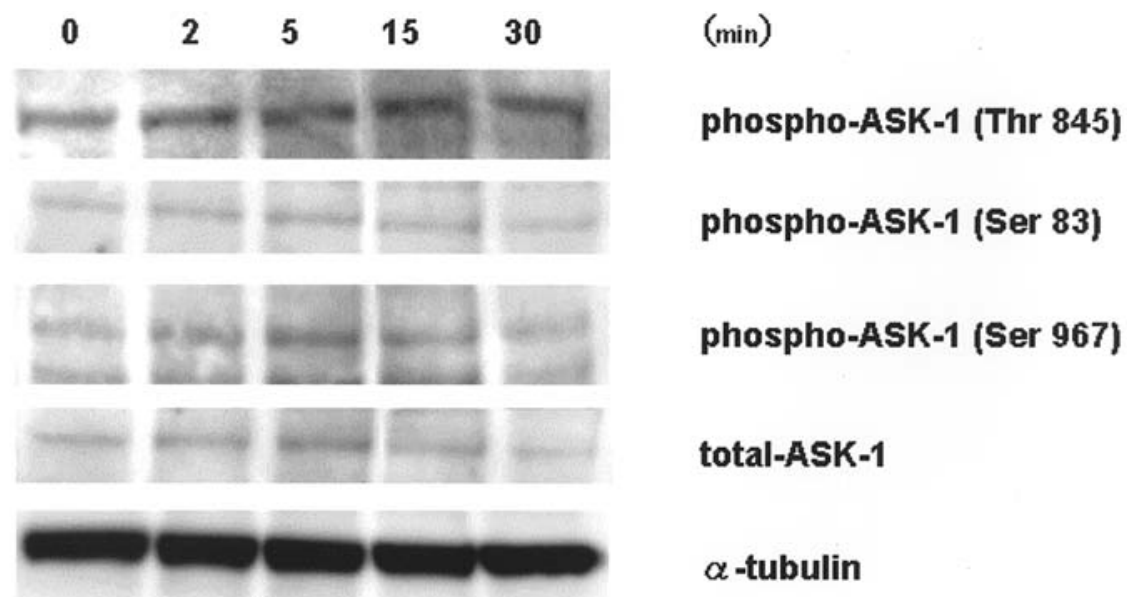

Figure 1. Time dependency of ASK-1 phosphorylation in HT-29 cells stimulated by FBS (1\%). HT-29 cells in serum-free media for 24 h were stimulated by FBS (1\%) and collected 0,2,5, 15, or 30 min after stimulation. Western blot analysis was performed as described in Materials and methods. Thirty micrograms of each sample was applied. Antibodies to phosphorylated-ASK-1 (Thr 845, Ser 83, Ser 967), total-ASK-1, and $\alpha$-tublin (for loading control) were used in Western blot analysis.

(A)

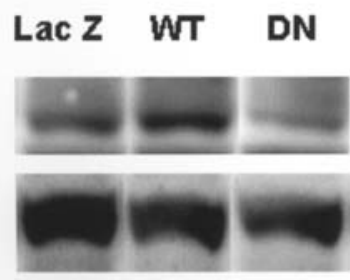

Serum stimulation (5 min)
(B)

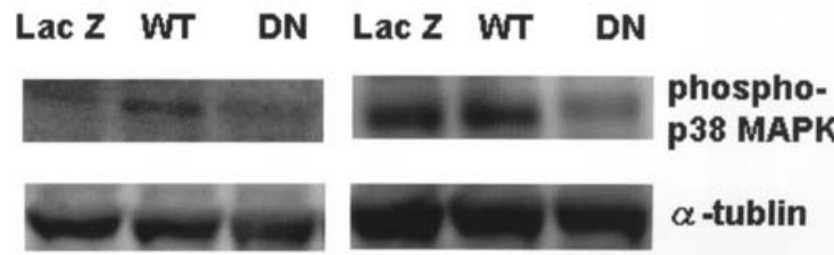

Day 1

Figure 2. Efficiency of DN-ASK-1 transfection for p38 MAPK phosphorylation. (A) HT-29 cells transfected with 100 MOI of Ad-LacZ, Ad-WT-ASK-1, or Ad-DN-ASK-1 were serum-starved and collected 5 min after FBS (1\%) stimulation. (B) HT-29 cells, incubated under the same condition, were transfected with Ad-LacZ, Ad-WT-ASK-1, or Ad-DN-ASK-1 at 100 MOI. After 24 and $48 \mathrm{~h}$ of transfection, the cells were collected and used for Western blot analysis. Western blot analysis was performed as described in Materials and methods. Thirty micrograms of each sample was applied. Antibodies to phospho-p38 and $\alpha$-tublin (for loading control) were used in Western blot analysis. (A) 24 h later (B) 48 h later, upper lane: p-p38 MAPK, lower lane: $\alpha$-tublin.

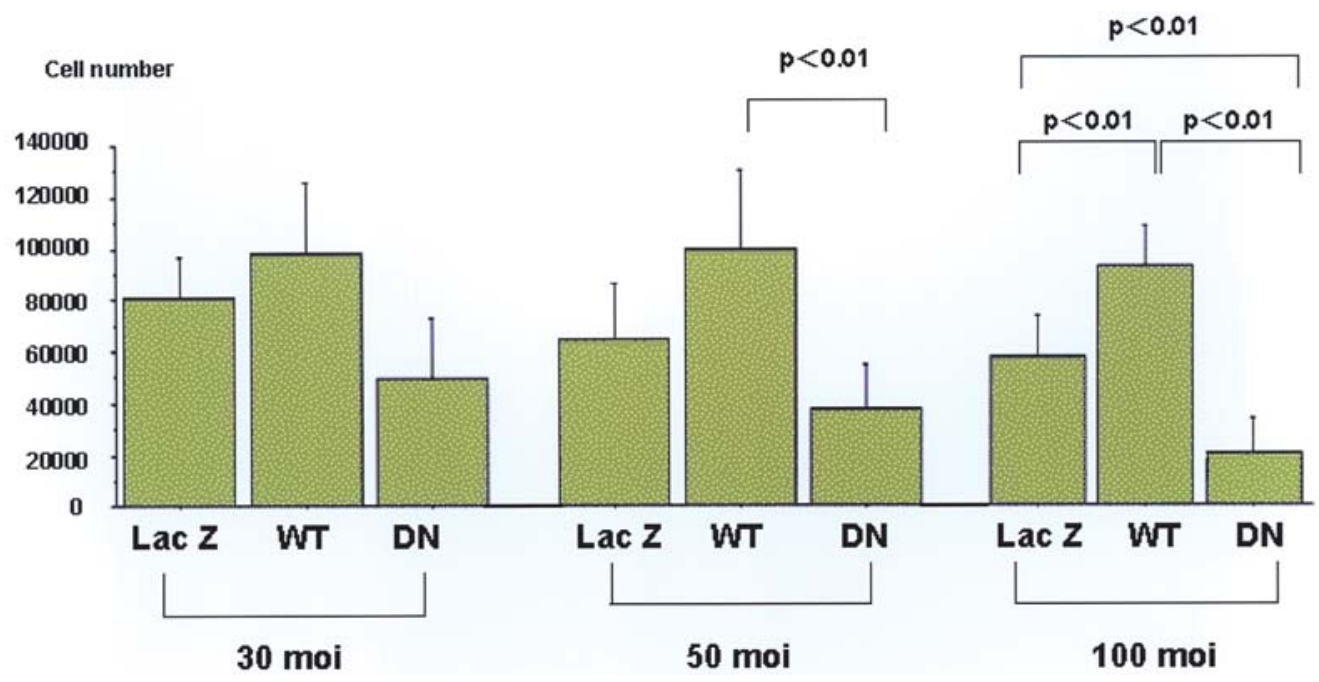

Figure 3. Efficiency of DN-ASK-1 transfection for viable cell number of HT-29 cells. HT-29 cells were seeded on 6-well cultured plates. They were transfected with Ad-LacZ, Ad-WT-ASK-1, Ad-DN-ASK-1 at 30, 50, 100 moi, cultured with $1 \%$ FBS for 5 days. Viable cell number was counted by a coulter counter. Each value represents the mean \pm SEM, (n=8); (a) 30 moi, (b) 50 moi, (c) 100 moi: p<0.01 vs. Ad-LacZ. 
Lac Z

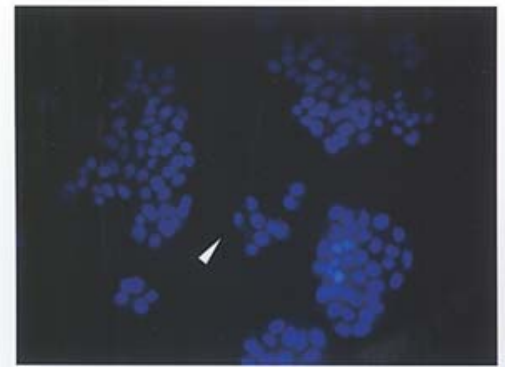

WT

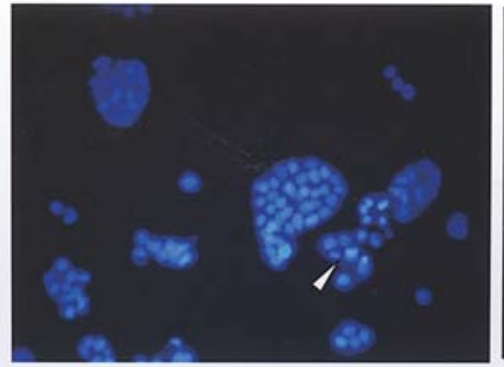

DN

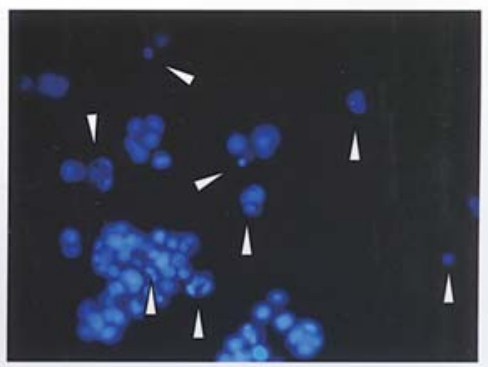

Figure 4. Detection of apoptotic cells after DN-ASK-1 transfection. HT-29 cells, cultured under the same conditions as described for Fig. 2, were transfected with Ad-LacZ, Ad-WT-ASK-1, or Ad-DN-ASK-1 at 100 MOI. After 24 h of transfection, the cells were stained with Hoechst 33342 (white arrows indicate apoptotic cells). LacZ, Ad-LacZ; WT, Ad-WT-ASK-1; DN, Ad-DN-ASK-1.

A

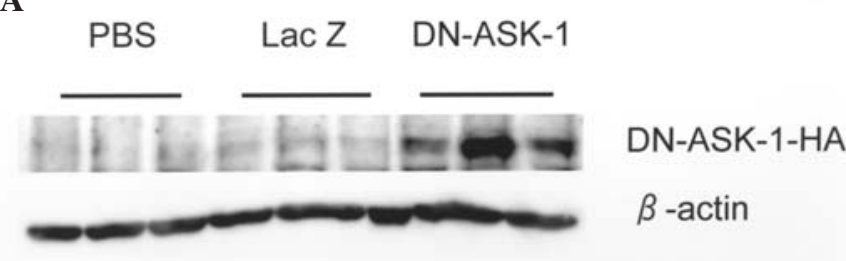

B

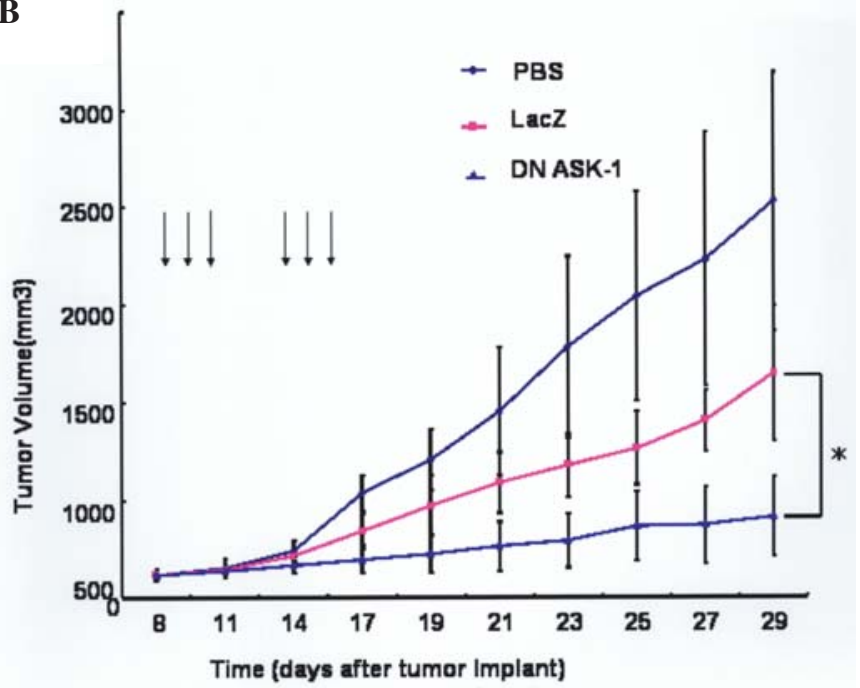

Figure 5. Confirmation of transfection of Ad-DN-ASK-1 into tumor xenografts of HT-29 cells and effect of subcutaneous injection of Ad-DNASK-1 on tumor xenografts in vivo. (A) Extracted proteins from HT-29 cell tumors were prepared for Western blot analysis $48 \mathrm{~h}$ after the last injection. Western blot analysis was performed as described in Materials and methods. Thirty micrograms of each sample was applied. Antibodies to HA-7 and $\beta$-actin (for loading control) were used in Western blot analysis. (B) Established tumors $\left(150 \mathrm{~mm}^{3}\right)$ were randomly assigned to three groups in a blinded manner and treated with Ad-LacZ (3x10 $10^{8}$ i.f.u.), Ad-DN-ASK-1 ( $3 \times 10^{8}$ i.f.u.), or PBS (vehicle control). Each treatment was performed from days 8 to 10 and from days 14 to 16 . Tumors were measured in 2 dimensions. Tumor volumes were calculated as described in Materials and methods. Each value represents the mean $\pm \mathrm{SEM},(\mathrm{n}=8)$. $\mathrm{P}<0.05$ vs. Ad-LacZ.

X-gal assay (12). Infection with Ad-LacZ at MOI of 100 was sufficient to transfer the gene to HT-29 cells. Transfection with Ad-DN-ASK-1 inhibited the serum-induced phosphorylation of p38 MAPK, a downstream molecule of ASK-1, while gene transfer of Ad-WT-ASK-1 induced such phosphorylation (Fig. 2A). To evaluate the continuous efficacy of Ad-DNASK-1, as well as the transient efficacy as shown in Fig. 2A, we analyzed the activation of p38 MAPK on days 1 and 2 after transfection. Ad-DN-ASK-1 inhibited the activation of p38 MAPK in HT-29 cells. However, Ad-WT-ASK-1 induced its activation (Fig. 2B). These findings showed that our constructed Ad-DN-ASK-1 inhibited the ASK-1-p38 MAPK pathway.

Effect of ASK-1 gene transfer on viable cell number of HT-29 cells. To examine the biological functions of ASK-1, we investigated the effect of Ad-DN-ASK-1 on cell proliferation. Viable cell number after treatment with Ad-WT-ASK-1 was increased as compared with that of control (treated with Ad-LacZ). In addition, transfection with ASK-1 and $\Delta \mathrm{N}$, constitutively active mutant of ASK-1, also increased the viable cell number similarly to Ad-WT-ASK-1 (data not shown). In contrast, the viable cell number after treatment with Ad-DN-ASK-1 was decreased as compared with that of control (Fig. 3). These changes in the number of HT-29 cells were affected in a dose (MOI)-dependent manner of transfection. We confirmed the reproducibility of these results in three separate experimental sessions.

Detection of apoptotic cells after ASK-1 transfection. To investigate the mechanism(s) responsible for the inhibition of HT-29 cell proliferation by Ad-DN-ASK-1, we focused on the association with apoptosis. The number of positive cells for Hoechst staining was significantly higher after Ad-DN-ASK-1 transfection than after Ad-LacZ transfection or Ad-WTASK-1-transfection (Fig. 4).

In vivo transfection of $A d-D N-A S K-1$ into tumor xenografs and its inhibitory effect on tumor growth. As shown in Fig. 5A, we first confirmed the specific expression of the HA-tag originating from Ad-DN-ASK-1-HA in the Ad-DN-ASK-1 treated tumors but not Ad-LacZ treated tumors. DN-ASK-1 protein was successfully expressed in tumor xenografts of HT-29 cells after local injection into the tumors. To assess the effect of Ad-DN-ASK-1 on the growth of tumor xenografts, 


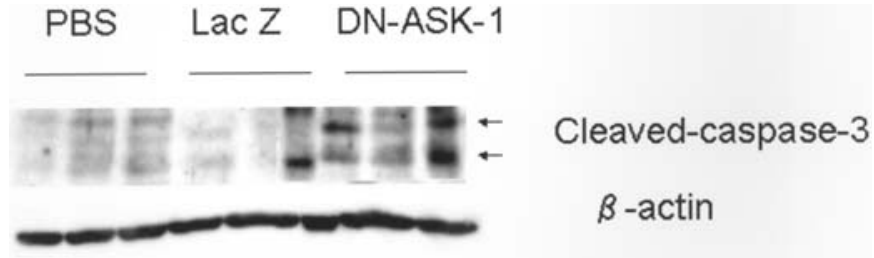

Figure 6. Expression of caspase-3 in the tumors xenografts of HT-29 cells after transfection with Ad-DN-ASK-1. Extracted proteins from HT-29 cell tumors were prepared for Western blot analysis $48 \mathrm{~h}$ after the last injection. Western blot analysis was performed as described in Materials and methods. Thirty micrograms of each sample was applied. Antibodies to cleavedcaspase-3 and $B$-actin (for loading control) were used in Western blot analysis.

we measured tumor volume after the local injection of Ad-DNASK-1 (3x108 i.f.u.), Ad-LacZ ( $3 \times 10^{8}$ i.f.u.), or PBS (vehicle control). On day 29 after inoculation, the volume of tumors treated with Ad-DN-ASK-1 was significantly smaller than that of tumors treated with Ad-LacZ or PBS (Fig. 5B).

Activation of caspase-3 in tumor xenografts of HT-29 cells by $A d-D N-A S K-1$. Caspase-3 was activated in tumor xenografts of HT-29 cells by two injections of Ad-DN-ASK-1. In contrast, neither Ad-LacZ nor PBS affected caspase-3 enzymatic activity (Fig. 6).

\section{Discussion}

We demonstrated that a recombinant adenovirus containing the dominant-negative mutant of ASK-1 (Ad-DN-ASK-1) inhibited the activation of p38 MAPK, a downstream signal of ASK-1, and suppressed the proliferation of HT-29 cells by apoptotic mechanisms in in vitro experiments. Tumor volume was most likely reduced by apoptotic mechanisms after transfection with Ad-DN-ASK-1 in in vivo experiments. To our knowledge, this is the first evidence suggesting that ASK-1 is involved in the proliferation of HT-29 cells and that Ad-DN-ASK-1 gene transfer might be useful for the treatment of colon cancer.

ASK-1 is one of the molecules located in MAPKKK, directly responsible for phosphorylation of MKK-3, MKK-6, SEK-1, and MKK-7, as well as activation of the p38 MAPK and JNK pathways $(2,7,14,15)$. ASK-1 is generally activated by oxidative stress, inflammatory cytokines such as TNF or IL-1ß, or calcium irritation, and then transmits signals to the MAPK pathway located downstream (3-6). ASK-1-p38 MAPK/JNK cascades regulate AP-1 activation in NOstimulated bronchial epithelial cells under some inflammatory conditions (13). Moreover, antioxidants such as thioredoxin, a physiological inhibitor of ASK-1, inhibit cytokine- and stress-induced apoptosis (3-6). Cardiac myocyte hypertrophy, a characteristic of cardiac remodeling, requires activation of ASK-1 and nuclear factor $\kappa \mathrm{B}(16)$. Thus, ASK-1 is considered essential for a variety of physiological functions, such as apoptosis (7). In gastric and colonic epithelial cells, JNK and p38 MAPK, downstream signals of ASK-1, are also activated by various external stimuli (17-19).

We previously reported that IL-1ß induces MAPK activation, including extracellular signal-regulated kinases
(ERK), JNK, and p38 MAPK, in normal rat gastric epithelial cells (RGMI cells) and weakly inhibits cell proliferation (20). In contrast, serum stimulation (2\%) induces phosphorylatedERK, -JNK, and -p38 MAPK as well as the expression of cyclooxygenase-2, a potent proliferative factor for gastrointestinal normal and cancer cells, in MKN 45 cells (gastric cancer cell line) and HT-29 cells (21). Thus, the role of ASK-1 linked with MAPK activation remains controversial with regard to the proliferation of gastrointestinal epithelial cells. Moreover, data on the proliferation and apoptosis of colon cancer cells are lacking. Phosphorylation of ASK-1 requires a threonine residue (Thr 845 ), present in a locus called the activation loop in the kinase domain $(22,23)$, and phosphorylation of Ser 967 and Ser 83 leads to inactivation of ASK-1 $(24,25)$. We first investigated activation of these three sites to evaluate the role of ASK-1 in proliferation of colon cancer (HT-29) cells after serum stimulation. Phosphorylation of ASK-1 at Thr 845 was markedly induced but not at sites of Ser 83 or Ser 967 . This finding suggested that phosphorylation of ASK-1 was closely related to HT-29 cell proliferation. To evaluate the implications of these findings, our previously reported gene transfer technique using DNASK-1 was performed, since no agent is available for the selective inhibition of ASK-1 activity $(2,12,26)$. Ad-DNASK-1-induced blockade of the downstream signal transmission of p38 MAPK and inhibition of cell proliferation reconfirmed that DN-ASK-1 was efficiently transfected into cells in our system and that ASK-1 has an important role in the proliferation of HT-29 cells. To elucidate underlying mechanism(s), we measured the number of apoptotic cells by Hoechst staining. Apoptotic cells increased only after the treatment with Ad-DN-ASK-1, but not after treatment with Ad-WT-ASK-1 or LacZ. Collectively, these findings suggested that ASK-1 was involved at least in cellular proliferation of HT-29 cells and that blockade of ASK-1 inhibited cell proliferation by causing apoptosis.

We next examined the efficacy of DN-ASK-1 gene transfer in an animal model of colon cancer to determine whether DNASK-1 inhibits tumor cell proliferation in vivo. We confirmed that DN-ASK-1 protein was successfully expressed in tumor xenografts of HT-29 cells. Moreover, repeated treatment with Ad-DN-ASK-1 significantly decreased the volume of the tumor xenografts as compared with vehicle-treated controls and adenoviral vector (LacZ)-treated controls. However, consistent with the results of previous studies, including ours (12), there was a faint nonspecific effect of control adenovirus on the tumor volume. Furthermore, DN-ASK-1 induced activation of apoptosis-related enzyme (caspase-3) in the treated tumors. These findings suggested that inhibition of ASK-1 activity participated in anti-tumor effects mediated by apoptosis both in in vivo and in vitro systems. A potential advantage of Ad-DN-ASK-1 transfection was suggested by the apparent lack of adverse effects, such as mortality or body weight or appetite loss. Therefore, the obtained initial evidence suggested that Ad-DN-ASK-1 gene transfer can significantly inhibit the growth of colon cancer and may thus be a new strategy for treatment. However, neither DN-ASK-1 nor WT-ASK-1 had any effect on the other two colon cancer cell lines: Caco2 (moderately differentiated adenocarcinoma cells) and Colon26 (highly potent cells for metastasis to liver 
or lung) cells. Our preliminary studies indicate that serum stimulation does not induce the activation of ASK-1 in either of these cell lines (unpublished data). In addition to differences in the histological features or genetic properties of colon cancer cells, whether or not ASK-1 activation is required for cell proliferation may be one reason why these three cell lines have different responses to DN-ASK-1. However, clinically, most colorectal cancers $(>70 \%)$ consist of well-differentiated adenocarcinoma cells, similar to HT-29 cells. DN-ASK-1 may therefore be useful in the clinical treatment of most, but not all, colon cancers. In addition, more efficient systems for the loco-regional delivery of target genes should be established, not only to enhance anti-tumor efficacy but also to avoid nonspecific responses. Further investigations are thus needed.

In conclusion, we obtained initial evidence suggesting that transfer of a dominant-negative mutant of the ASK-1 gene inhibited colon cancer growth in vitro and in vivo. Our study may provide new insights into the molecular mechanisms of colorectal cancer.

\section{Acknowledgements}

This study was supported in part by a Grant-in-Aid for Scientific Research from the Ministry of Education, Culture, Sports Science and Technology of Japan.

\section{References}

1. Tobiume K, Inage T, Takeda K, Enomoto S, Miyazono K and Ichijo H: Molecular cloning and characterization of the mouse apoptosis signal-regulating kinase 1. Biochem Biophys Res Commun 23: 9905-9910, 1997.

2. Ichijo H, Nishida E, Irie K, ten Dijke P, Saitoh M, Moriguchi T, Takagi M, Matsumoto K, Miyazono K and Gotoh Y: Induction of apoptosis by ASK1, a mammalian MAPKKK that activates SAPK/JNK and p38 signaling pathways. Science 27: 590-594, 1997.

3. Saitoh M, Nishitoh H, Fujii M, Takeda K, Tobiume K, Sawada Y, Kawabata M, Miyazono K and Ichijo H: Mammalian thioredoxin is a direct inhibitor of apoptosis signal-regulating kinase (ASK) 1. EMBO J 17: 2596-2606, 1998.

4. Nishitoh H, Saitoh M, Mochida Y, Takeda K, Nakano H, Rothe M, Miyazono K and Ichijo H: ASK1 is essential for JNK/SAPK activation by TRAF2. Mol Cell 2: 389-395, 1998.

5. Chang HY, Nishitoh H, Yang X, Ichijo H and Baltimore D: Activation of apoptosis signal-regulating kinase 1 (ASK1) by the adapter protein Daxx. Science 281: 1860-1863, 1998.

6. Takeda K, Matsuzawa A, Nishitoh H and Ichijo H: Roles of MAPKKK ASK1 in stress-induced cell death. Cell Struct Funct 28: 23-29, 2003.

7. Ichijo H: From receptors to stress-activated MAP kinases. Oncogene 18: 6087-6093, 1999.

8. Tobiume K, Matsuzawa A, Takahashi T, Nishitoh H, Morita K, Takeda K, Minowa O, Miyazono K, Noda T and Ichijo H: ASK1 is required for sustained activations of JNK/p38 MAP kinases and apoptosis. EMBO Rep 2: 222-228, 2001.

9. Takeda K, Hatai T, Hamazaki TS, Nishitoh H, Saitoh M and Ichijo H: Apoptosis signal-regulating kinase 1 (ASK1) induces neuronal differentiation and survival of PC12 cells. J Biol Chem 275: 9805-9813, 2000 .
10. Sayama K, Hanakawa Y, Shirakata Y, Yamasaki K, Sawada Y, Sun L, Yamanishi K, Ichijo H and Hashimoto K: Apoptosis signal-regulating kinase 1 (ASK1) is an intracellular inducer of keratinocyte differentiation. J Biol Chem 276: 999-1004, 2001.

11. Gryfe R, Swallow C, Bapat B, Redston M, Gallinger S and Couture J: Molecular biology of colorectal cancer. Curr Probl Cancer 21: 233-300, 1997.

12. Suto R, Tominaga K, Mizuguchi H, Sasaki E, Higuchi K, Kim S, Iwao $\mathrm{H}$ and Arakawa T: Dominant-negative mutant of c-Jun gene transfer: a novel therapeutic strategy for colorectal cancer. Gene Ther 11: 187-193, 2004.

13. Jibiki I, Hashimoto S, Maruoka S, Gon Y, Matsuzawa A, Nishitoh H, Ichijo H and Horie T: Apoptosis signal-regulating kinase 1-mediated signaling pathway regulates nitric oxideinduced activator protein-1 activation in human bronchial epithelial cells. Am J Respir Crit Care Med 167: 856-861, 2003.

14. Davis RJ: Signal transduction by the JNK group of MAP kinases. Cell 13: 239-252, 2000.

15. Chang L and Karin M: Mammalian MAP kinase signalling cascades. Nature 1: 37-40, 2001.

16. Omura T, Yoshiyama M, Kim S, Matsumoto R, Nakamura Y, Izumi Y, Ichijo H, Sudo T, Akioka K, Iwao H, Takeuchi $\mathrm{K}$ and Yoshikawa J: Involvement of apoptosis signal-regulating kinase-1 on angiotensin II-induced monocyte chemoattractant protein-1 expression. Arterioscler Thromb Vasc Biol 24: 270-275, 2004

17. Mitsuyama K, Tsuruta O, Matsui Y, Harada K, Tomiyasu N, Suzuki A, Takaki K, Masuda J, Handa K, Satoh Y, Bennett BL, Toyonaga A and Sata M: Activation of c-Jun N-terminal kinase (JNK) signalling in experimentally induced gastric lesions in rats. Clin Exp Immunol 143: 24-29, 2006.

18. Krueger S, Hundertmark T, Kalinski T, Peitz U, Wex T, Malfertheiner P, Naumann M and Roessner A: Helicobacter pylori encoding the pathogenicity island activates matrix metalloproteinase 1 in gastric epithelial cells via JNK and ERK. J Biol Chem 281: 2868-2875, 2006

19. Hundley TR and Rigas B: Nitric oxide-donating aspirin inhibits colon cancer cell growth via mitogen-activated protein kinase activation. J Pharmacol Exp Ther 316: 25-34, 2006.

20. Tominaga K, Arakawa T, Tsuno M, Kim S, Iwao H and Kuroki T: Increased mitogen-activated protein kinase activities stimulated with interleukin-1-beta and mechanism(s) of the kinase signaling pathways in rat gastric epithelial cells. Digestion 61: 30-38, 2000.

21. Tominaga K, Higuchi K, Sasaki E, Suto R, Watanabe T, Fujiwara Y, Oshitani N, Matsumoto T, Kim S, Iwao H and Arakawa T: Correlation of MAP kinases with COX-2 induction differs between MKN45 and HT29 cells. Aliment Pharmacol Ther (Suppl 1): 143-150, 2004.

22. Tobiume K, Saitoh M and Ichijo H: Activation of apoptosis signal-regulating kinase 1 by the stress-induced activating phosphorylation of pre-formed oligomer. J Cell Physiol 191: 95-104, 2002.

23. Gotoh Y and Cooper JA: Reactive oxygen species- and dimerization-induced activation of apoptosis signal-regulating kinase 1 in tumor necrosis factor-alpha signal transduction. J Biol Chem 10: 17477-17482, 1998.

24. Zhang L, Chen J and Fu H: Suppression of apoptosis signalregulating kinase 1-induced cell death by 14-3-3 proteins. Proc Natl Acad Sci USA 20: 8511-8515, 1999.

25 Kim AH, Khursigara G, Sun X, Franke TF and Chao MV: Akt phosphorylates and negatively regulates apoptosis signalregulating kinase 1. Mol Cell Biol 21: 893-901, 2001.

26. Chen Z, Seimiya H, Naito M, Mashima T, Kizaki A, Dan S, Imaizumi M, Ichijo H, Miyazono $\mathrm{K}$ and Tsuruo T: ASK1 mediates apoptotic cell death induced by genotoxic stress. Oncogene 18: 173-180, 1999. 